chemiluminescent microparticle immunoassay (CMIA, private laboratories). The non-treponemal Venereal Disease Research Laboratory (VDRL) assay was used to screen patients previously-treated for syphilis. An in-house PCR assay tested ulcerderived DNA extracts for TP. The study received Ethics Committee approval.

Results 93 primary syphilis cases (92 with ulceration, 1 with inguinal lymphadenopathy) were diagnosed in the study period. TP PCR was performed in 84/92 (91\%) cases and positive in $76 / 84$ (90\%) cases. Overall, syphilis serology detected 79/93 (85\%) primary syphilis cases. 75/93 (81\%) cases had no laboratory evidence of prior syphilis; 18 cases (19\%) had previous reactive treponemal serology. Treponemal antibodies were present in 63/75 (84\%) first-episode syphilis cases; $12 / 75$ (16\%) had non-reactive treponemal serology. Most cases with previously-treated syphilis had reactive VDRL tests $(16 / 18,89 \%)$. There were 64 PCR positive/serology reactive cases, 12 TP PCR positive/serology non-reactive cases, 7 PCR negative/serology reactive cases and one clinically-diagnosed case (negative TP PCR/non-reactive serology). Serology was reactive in all 9 cases where TP PCR was not performed (8 EIA reactive, 1 VDRL reactive).

Conclusion Primary syphilis is best diagnosed using a combined approach with TP PCR and syphilis serology.

\section{P357 A NEW MEASURE OF SEXUAL WELLBEING FOR COMMUNITY SURVEYS: DEVELOPMENT AND VALIDATION OF THE THE NATSAL-SW}

${ }^{1} \mathrm{~K}$ Mitchell ${ }^{*},{ }^{2} \mathrm{M}$ Palmer, ${ }^{1} \mathrm{R}$ Lewis, ${ }^{1} \mathrm{R}$ Boso Perez, ${ }^{1} \mathrm{~K}$ Maxwell, ${ }^{2} \mathrm{~W}$ Macdowell, ${ }^{2} \mathrm{D}$ Reid, ${ }^{2} \mathrm{C}$ Bonell, ${ }^{3} \mathrm{P}$ Sonnenberg, ${ }^{3} \mathrm{C}$ Mercer, ${ }^{4} \mathrm{~J}$ Fortenberry. ${ }^{1}$ University of Glasgow, Glasgow, UK; ${ }^{2}$ London School of Hygiene and Tropical Medicine, London, UK; ${ }^{3}$ University College London, London, UK; ${ }^{4}$ Indiana University, Indianapolis, USA

\subsection{6/sextrans-2021-sti.401}

Background Sexual wellbeing is intrinsic to public health but long-standing conflation of sexual health and sexual wellbeing has limited our ability to address everyday sexual issues. This study proposed a seven-domain model, and developed and validated a brief measure for community surveys.

Method Domains of sexual wellbeing were determined through critical engagement with wide-ranging literature and 40 semi-structured interviews to explore resonance with lived experiences. Measure development involved 7 cognitive interviews and two web-based surveys of general population samples $(n=590, n=814)$, to assess performance of individual items, conduct exploratory and confirmatory factor analysis, and examine whether the resultant measure was associated with external variables as hypothesised. A sub-sample $(n=113)$ completed the survey again after two weeks to test re-test reliability.

Results We proposed seven domains of sexual wellbeing: security and safety; respect; self-esteem; resilience; forgiveness of past sexual experiences; self-determination and comfort. Semistructured interviews confirmed the relevance of these domains to lived experiences of sex and sexuality. Drawing on the semi-structured and cognitive interviews we drafted a 25-item measure to capture these domains. Based on individual item assessment and exploratory and confirmatory factor analyses, we trimmed the measure to 13 -items. The confirmatory factor analysis indicated that a 'general specific model' had best fit (RMSEA: 0.064; CFI: 0.975, TLI: 0.962), and functioned equivalently across age groups, genders, sexual orientation and relationship status. The final measure was associated with external variables in the directions hypothesised (all $\mathrm{p}<0.001$ ), including sexual functioning (coefficient $=0.924$ ), mental wellbeing (0.454), self-esteem (0.564), sexual esteem (0.563), body image $(0.232)$, depression $(-0.384)$, anxiety $(-0.340)$, sexual satisfaction (0.680) and sexual distress (-0.615) and demonstrated good test-retest reliability (ICC $=0.78$ ).

Conclusion Our conceptual model and 13-item measure distinguishes sexual wellbeing from sexual health and enables sexual wellbeing to be quantified and understood within and across populations.

\section{P358 ASSESSING SEXUAL HEALTH SERVICES AT A PUBLIC UNIVERSITY IN THE UNITED STATES}

A Camino*, M Whitfield, N Van Wagoner. University of Alabama At Birmingham School of Medicine, Birmingham, USA

\subsection{6/sextrans-2021-sti.402}

Background The American National College Health Association reports that college students have frequent, condomless sex. Student health and wellness clinics (SHWC) offer sexual health services, but few have dedicated sexual health clinics (SHC). We evaluated screening service use at a university SHWC after implementation of a SHC two half-days per week.

Methods This was a retrospective analysis of data from patients receiving sexual health screening at the University of Alabama at Birmingham (UAB) SHWC from 2015 to 2019. Demographic variables and rates of STIs were extracted from the electronic medical record and were compared by clinic (SHC vs. SHWC). Univariate models were fit, and multi-variable models will be fit, selecting variables with $\mathrm{p}$ values of 0.1 or less. Odds ratios with corresponding 95\% confidence intervals for univariate analysis are presented.

Results A total of 5025 STI screenings were performed. Males (OR 4.13; 3.61-4.72), undergraduates (OR 1.33; 1.15-1.54), and persons reporting sex with the same sex (OR 1.88; $1.56-$ 2.28), were significantly more likely to seek care at the SHC. Students with symptoms were more likely to seek care at the SHWC (OR 0.53; 0.47-0.61), while persons who reported contact with STIs were more likely to seek care at the SHC (OR 2.88; 2.22-3.74). The overall percentage of positive screenings was $9.3 \%$ for chlamydia (CT), 3.0\% for gonorrhea (GC), $0.8 \%$ for trichomoniasis (TV), $0.7 \%$ for syphilis, and $0.3 \%$ for HIV with higher percentages of positive for CT (OR 1.60; 1.30-1.96) and GC (OR 2.02; 1.44-2.85) in the SHC.

Conclusion Embedding a dedicated SHC within a university SHWC may expand populations reached for STI screening. With higher percentages of patients testing positive for CT and GC, a SHC may allow for greater diagnosis and treatment of STIs in general screening and persons presenting as contacts.

\section{P359 OPTIMIZED METHODS TO MODEL MYCOPLASMA GENITALIUM REPRODUCTIVE TRACT INFECTION IN PIG-TAILED MACAQUES}

L Aguila*, D Patton, G Wood. University of Washington, Seattle, USA 